\title{
Behavior Selection and Memory-based Learning for Artificial Creature Using Two-layered Confabulation
}

\author{
Se-Hyung Cho ${ }^{1}$, Ye-Hoon Kim ${ }^{1}$, In-Won Park ${ }^{1}$, and Jong-Hwan Kim ${ }^{1}$, Senior Member, IEEE \\ ${ }^{1}$ Department of Electrical Engineering and Computer Science \\ Korea Advanced Institute of Science and Technology (KAIST) \\ 373-1 Guseong-dong, Yuseong-gu, Daejeon 305-701, Republic of Korea, \\ e-mail : (shcho, yhkim, iwpark, johkim)(a)rit.kaist.ac.kr
}

\begin{abstract}
Confabulations, where millions of items of relevant knowledge are applied in parallel in the human brain, are typically employed in thinking. This paper proposes a novel behavior selection architecture and memory-based learning method for an artificial creature based on two-layered confabulation. A behavior is selected by considering both internally generated will and the context of the external environment. Proposed behavior selection using a confabulation scheme is a parallel process in which a number of behaviors are considered simultaneously. An arbitration mechanism is employed to choose a proper behavior which is to be put into an action. Also memory-based behavior learning is proposed, where the memory has the selection probabilities of behaviors based on will and context. The learning module updates the contents of memory according to the user-given reward or penalty signal. To demonstrate the effectiveness of the proposed scheme, an artificial creature is implemented in the $3 \mathrm{D}$ virtual environment such that it can interact with a human being considering its will and context.
\end{abstract}

\section{INTRODUCTION}

Many service robots and entertainment robots are already well-known to help in simple housework and to share friendship with the user. Software pet-type robots, which imitate animal's spontaneity, are developed to be mounted into actual hardware platform or electronic device, i.e. cell-phone or computer. An artificial creature can be used as an intermediate interface for interactions between human and service robot. It should hold outward appearances and knowledge including behavior patterns so that it can resemble a living creature and approach to the user with familiarity. In this sense, it requires confabulation, which imitates a human thought process, and the context-awareness in behavior selection. In addition, it should continuously adapt to the varying environment or to the user's preference. Thus, the learning process is needed like a real creature's adaptation ability.

There are researches which mimic a creature's externally observed functions, and analyze a human's brain structure and thought mechanism in physiological perspectives. Confabulation as a thought mechanism is a process of making a plausible 'spurious' memory from inexperienced facts in the brain using similar reminiscences in the past. Through this process, humans can generalize the past experiences and cope with the indirectly experienced situations [1]-[3].

In this paper, a behavior selection architecture based on two-layered confabulation is proposed. The architecture is composed of two parallel layers in order to consider both internally generated will and the context of the external environment. Each behavior selection layer suggests will-based behaviors and context-based behaviors, individually. Finally, an adequate behavior considering both internal state and context is chosen to put into an action. Cogent confabulation is employed to choose an adequate behavior based on cogency in behavior selection. In cogent confabulation, many conclusion symbols are considered at the same time and the best likely symbol is projected as a conclusion [1]. All contexts, internal states and behaviors are represented as symbols and the knowledge is represented by each symbol's link in the proposed architecture.

Furthermore, memory-based behavior selection is proposed. The memory module holds the probability information which represents the plausibility of each behavior corresponding to the will or context. The probability information is used in confabulation process. Since the artificial creature contains a memory module [4]-[10], behaviors can be learned according to the changed situation. Memory-based behavior learning is accomplished by two steps. The first step provides all the memory contents through an expert's hand as their initialization on plausible behaviors of the artificial creature. The second step is the memory-based reinforcement learning using user's reward or penalty to adapt to the user's preference. The effectiveness of the proposed scheme is demonstrated by implementing an artificial creature in the $3 \mathrm{D}$ virtual environment which can interact with a human being considering its will and context. This paper is organized as follows: Section II presents essential terms, which are required to embody an artificial creature, are defined including overall architecture of artificial creature and the basic idea of confabulation theory. Section III and Section IV describe the behavior selection based on two-layered confabulation and memory-based learning method, respectively. Section V describes the experimental results of artificial creature in virtual environment The concluding remarks and future works follow in Section VI.

\section{TWO-LAYERED CONFFABULATION ARCHITECTURE}

\section{A. Architecture for Artificial Creature}

In general, the architecture for artificial creature mimics a decision mechanism of the real living creature, which faces a certain situation and considers its own desire to make a decision for a proper behavior. Fig. 1 shows a typical architecture, where a behavior is selected by the behavior system 
considering the perception and motivation [11]-[24]. By using perception system, the artificial creature finds out the situation of external environment. Motivation system has an influence on the behavior system for a proper behavior selection. The resulting behavior gets expressed through the motor system. Thus, it requires an adequate behavior selecting architecture between the perception system and the motor system.

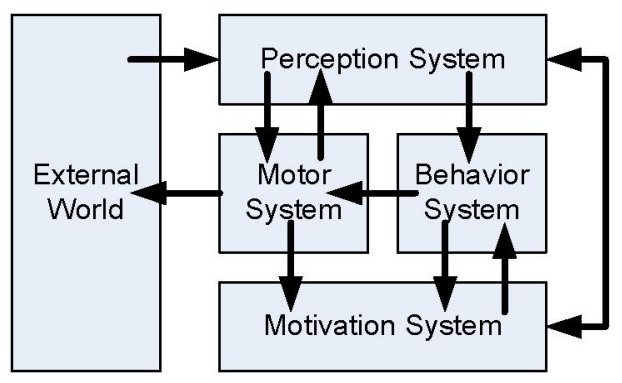

Fig.1 A schematic diagram of a situated character

To activate a behavior similarly as the real creature, the behavior system should reflect the internal state such as motivation, homeostasis and emotion. Also, as the environment continuously changes, the context of external environment should be considered in order to select an appropriate behavior. If the architecture is based on the priority between behaviors as shown in Fig. 2, the behavior based on the external environment constrains the behavior based on the internal state [12], [13]. Thus, it is impossible to realize a deliberative behavior, which considers both external and internal states at the same time. Consequently, an architecture incorporating both internal state and external environment is necessary.

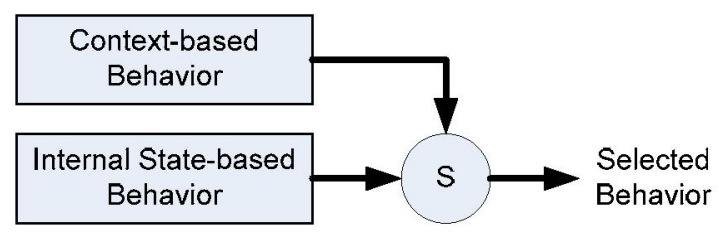

Fig. 2 Conventional behavior selector based on the behavior priority

\section{B. Two-Layered Confabulation Architecture}

The proposed architecture selects a behavior based on cogency of two-layered confabulations, which integrates the internal state and context. It is expected to realize more natural behavior by using this architecture rather than the simple architecture with the priority.

In the proposed architecture as shown in Fig. 3, both internal state and context information are used for behavior selection, referring to the memory module which holds the probability information representing the plausibility of each behavior corresponding to the will or context. The probability information is employed in confabulation process. Since the architecture contains a memory module, behaviors can be learned according to the changed situation.

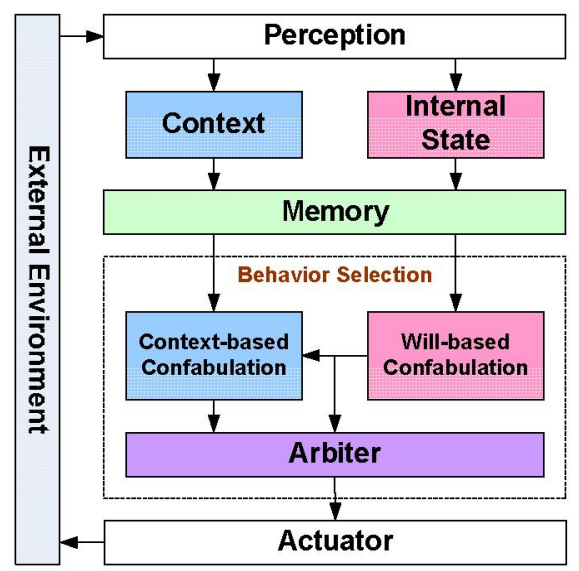

Fig. 3 The two-layered architecture for artificial creature

Behavior selection module is divided into three sub-modules: will-based confabulation, context-based confabulation, and an arbiter. In the will-based confabulation sub-module, most suitable behaviors are recommended by calculating cogency using the current internal states. The context-based confabulation sub-module calculates cogency of the recommended behaviors by considering the external context. Finally, the arbiter chooses a fittest behavior using the probabilities of the behaviors calculated by both will-based confabulation and context-based confabulation. That is, the resulting behavior is being recommended through the confabulation process. Using this architecture, the artificial creature can show a behavior satisfying its own desires and fitted to the external environment.

\section{Confabulation Theory}

Bayesian and cogent confabulation techniques are used to represent formal logic for inductive reasoning. These two methods reason by using the confidence to all conclusions. In the inductive reasoning, if the probability of $a, b, c$, and $d$ are given as a partition of a sample space $S$ and suppose that event $E$ occurs, then the confidence of event $E$ occurring is represented by using Bayesian posterior probability, $p(e \mid a b c d)$. In cogent confabulation, on the other hand, the confidence of conclusion is represented as cogency, $p(a b c d \mid e)$. Cogency can be calculated using Bayes' theorem as follows [1], [2]:

$$
\begin{aligned}
p(a b c d \mid e)^{4}= & {[p(a b c d e) / p(a e)] \cdot[p(a b c d e) / p(b e)] . } \\
& {[p(a b c d e / p(c e))] \cdot[p(a b c d e) / p(d e)] . } \\
& {[p(a \mid e) \cdot p(b \mid e) \cdot p(c \mid e) \cdot p(d \mid e)] }
\end{aligned}
$$

First four probabilities can be approximated as a constant number in any given situations. In general, these assumptions are plausible approximations as follows:

$$
\begin{aligned}
& {[p(a b c d e) / p(a e)] \cdot[p(a b c d e) / p(b e)] \cdot} \\
& \quad[p(a b c d e) / p(c e)] \cdot[p(a b c d e) / p(d e)] \approx K \\
& p(a b c d \mid e)^{4} \approx K \cdot[p(a \mid e) \cdot p(b \mid e) \cdot p(c \mid e) \cdot p(d \mid e)]
\end{aligned}
$$

where $K$ is a constant.

Once the first four probabilities are considered as a constant, 
having the maximum cogency is equivalent to maximizing the probability, $\left[p(a \mid e)^{\cdot} p(b \mid e)^{\cdot} p(c \mid e)^{\cdot} p(d \mid e)\right]$ [1], [2]. This process is known as confabulation. In this paper, the confabulation process is applied to artificial creature's behavior selection.

\section{BEHAVIOR SELECTION}

\section{A. Will-based Confabulation}

In the confabulation theory, words in the lexicon are assumed fact symbol and a suitable word to given phrase is conclusion [1]. Inspired by this mechanism, behavior selection is achieved by confabulation. The set of assumed fact corresponds to the artificial creature's will or the context of the environment. And conclusion set corresponds to the artificial creature's behavior set. A lot of assumed facts of will-based confabulation are generated from the artificial creature's internal states such as motivation, homeostasis and emotion. The confabulation process requires the behavior probability to each of the internal states, which is preserved in the memory module. The cogency of will-based confabulation can be computed using Bayes' rule as follows:

$$
\begin{gathered}
E_{\text {will }}\left(b_{1}\right)=p\left(w_{1} \mid b_{1}\right) \cdot p\left(w_{2} \mid b_{1}\right) \cdots \cdots p\left(w_{m} \mid b_{1}\right) \\
E_{\text {will }}\left(b_{2}\right)=p\left(w_{1} \mid b_{2}\right) \cdot p\left(w_{2} \mid b_{2}\right) \cdots \cdot p\left(w_{m} \mid b_{2}\right) \\
\vdots \\
E_{\text {will }}\left(b_{l}\right)=p\left(w_{1} \mid b_{l}\right) \cdot p\left(w_{2} \mid b_{l}\right) \cdots \cdot p\left(w_{m} \mid b_{l}\right)
\end{gathered}
$$

where $E_{\text {will }}$ is defined as cogency value (expectation) of the behavior to the will. $b_{j}, j=1,2, \ldots, l$ represents $j$ th behavior in a behavior set. $p\left(w_{i} \mid b_{i}\right)$ is a conditional probability between $i$ th will and $j$ th behavior. $l$ and $m$ represents the number of behaviors and the number of wills, respectively. Once the cogency values between the behaviors and their will are calculated, the behaviors with highest cogency values are recommended to the context-based confabulation. Since most of the animals load up to four objects into working memory at one time [25], similar number of behaviors can be recommended.

\section{B. Context-based Confabulation}

A lot of assumed facts of context-based confabulation are generated from the external environment such as time, place and object. In this paper, when, where and what (time, place and objects) are considered as contexts in a given situation. The cogency of context-based confabulation can be similarly calculated using Bayes' rule as follows:

$$
\begin{gathered}
E_{\text {context }}\left(b r_{1}\right)=p\left(c_{1} \mid b r_{1}\right) \cdot p\left(c_{2} \mid b r_{1}\right) \cdots p\left(c_{n} \mid b r_{1}\right) \\
E_{\text {context }}\left(b r_{2}\right)=p\left(c_{1} \mid b r_{2}\right) \cdot p\left(c_{2} \mid b r_{2}\right) \cdots p\left(c_{n} \mid b r_{2}\right) \\
\vdots \\
E_{\text {context }}\left(b r_{k}\right)=p\left(c_{1} \mid b r_{k}\right) \cdot p\left(c_{2} \mid b r_{k}\right) \cdots p\left(c_{n} \mid b r_{k}\right)
\end{gathered}
$$

where $E_{\text {context }}$ is defined as cogency value (expectation) of the behavior to the context. $c_{i}, i=1,2, \ldots, n$ represents $i$ th context, $b r_{j}, j=1,2, \ldots, k$ represents $j$ th recommended behavior from the will-based confabulation. $p\left(c_{i} \mid b r_{j}\right)$ is a conditional probability between $i$ th context and $j$ th recommended behavior. $k$ and $n$ represents the number of recommended behaviors and the number of contexts, respectively. The calculation is carried out for the recommended behaviors from the will-based confabulation to compute the adequacy reflecting environmental conditions.

\section{Arbiter}

The arbiter decides the final behavior based on the results of will-based confabulation, $E_{\text {will }}\left(b_{j}\right)$, and context-based confabulation, $E_{\text {context }}\left(b r_{j}\right)$. The behavior is determined by the max-product operation as follows:

$$
E_{\text {arbiter }}(b s)=\max \left[E_{\text {will }}\left(b r_{j}\right) \cdot E_{\text {context }}\left(b r_{j}\right)\right], \quad j=1, \cdots, k
$$

where $b s$ is the finally selected behavior.

The behavior having the highest value among the recommended behaviors is selected as the final output.

\section{MEMORY-BASED BEHAVIOR LEARNING}

As Fig. 4 shows, the memory module consists of context memory and internal state memory. Red arrows represent the link between the behavior and the context and internal state. The probability of the behavior which is related to the context and internal state is saved in the memory. For instance, a high probability value is memorized if eating behavior in the kitchen is adequate.

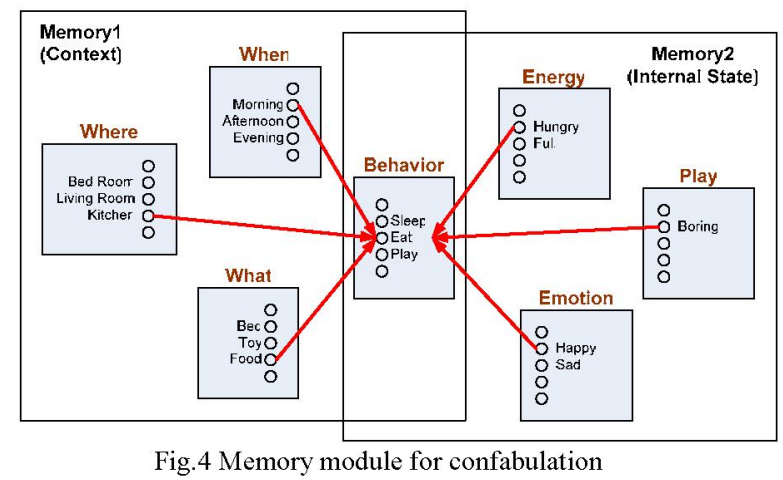

The memory-based learning technique guides the artificial creature's predefined behavior patterns into adaptive behaviors to survive in the new environment. This can be accomplished by two steps. The first step is to give all of the confabulation probabilities by an expert's hand as shown in Fig. 5. If incorrect probabilities are given, plausible behaviors cannot be expected and thus it requires careful settings. This step is used to set memory contents in the initialization process.

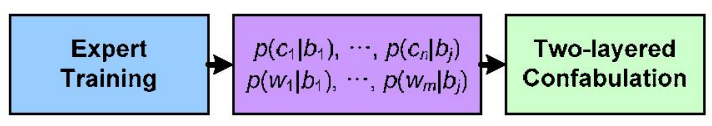

Fig. 5 Initial learning process by an expert

The second step is the memory-based reinforcement learning using feedback signals from changing environment as shown 
in Fig. 6. In the proposed structure, the user allows to grant either reward or penalty by patting or hitting an artificial creature to learn a desired behavior at a given situation.

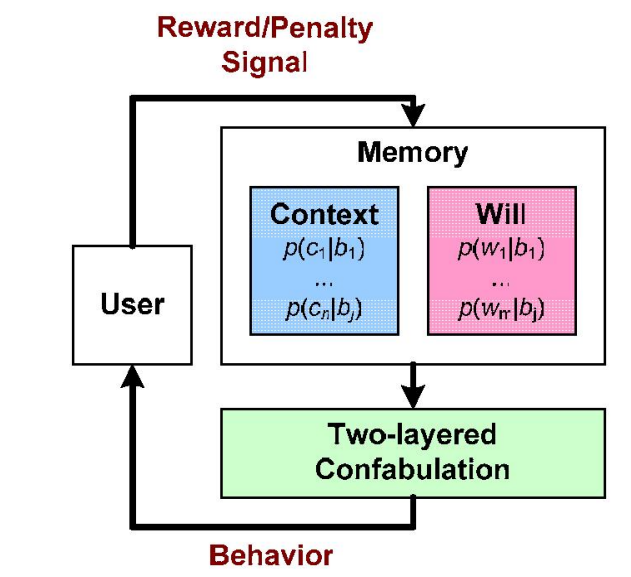

Fig. 6 Memory-based reinforcement learning using feedback

In order to improve the reinforcement learning, confabulation probability gets increased when patting input is given at a particular situation and vice versa for inducing hitting input as follows:

$$
p_{\text {temp }}\left(c_{i} \mid b_{j}\right)= \begin{cases}p_{t}\left(c_{i} \mid b_{j}\right)+\delta & \text { (if input is a reward) } \\ p_{t}\left(c_{i} \mid b_{j}\right)-\delta & \text { (if input is a penalty) } \\ p_{t}\left(c_{i} \mid b_{j}\right) & \text { (otherwise) }\end{cases}
$$

where $p_{\text {temp }}$ represents a temporal probability by interaction, $p_{t}\left(c_{i} \mid b_{j}\right)$ is a conditional probability between $i$ th context and $j$ th behavior, and $t$ and $\delta$ represent time and learning rate, respectively. There is a constraint that sum of all probabilities must be equaled to one.

$$
p_{t+1}\left(c_{i} \mid b_{j}\right)=\frac{p_{\text {temp }}\left(c_{i} \mid b_{j}\right)}{\sum_{i=1}^{l} p_{\text {temp }}\left(c_{i} \mid b_{j}\right)}
$$

By this technique, confabulation probability is adjusted such that the undesired behavior can be restrained from being activated.

\section{EXPERIMENTAL RESULTS}

\section{A. Objectives}

The goal of experiment is to create an artificial creature which behaves as per its will considering the context in a given situation. Context awareness enables it to carry out a proper decision in an unexpected environment. To achieve this purpose and to measure the performance of proposed architecture, the following desired scenarios among the behaviors, context and will are assumed.

i) Eating behavior would never be carried out around the toilet.

ii) Even though creature may not be hungry, eating behavior would be selected if the food is in the surroundings. iii) Even though creature is sleepy, sleeping behavior will not selected if the bed is not in the surroundings.

iv) If creature is too sleepy, sleeping behavior will be selected at any location.

\section{B. Experimental Environment}

The interactive learning method and the proposed behavior selection mechanism are applied to the synthetic character, 'Rity', which is implemented by OpenGL in a 3D virtual space. The sampling rate of the computational model is set to $0.1 \mathrm{sec}$. Rity has 12 DOFs, 46 sensor symbols and 73 behaviors. It is composed of perception module, internal state module, behavior selection module, learning module and motor module. More detailed descriptions are presented in [12]-[17].

Internal states of Rity are defined based on those of a real dog such as motivation, homeostasis and emotion. Parameter values from (3), (4) were set as follows: $n=2, m=8, k=3, l$ =40.: $n=3, m=8, k=3, l=40$. The assumed facts of will-based confabulation are classified in Table 1 .

TABLE. 1 ASSUMED FACTS OF WLL-BASED CONFABULATION
\begin{tabular}{|c|c|}
\hline $\begin{array}{c}\text { Internal } \\
\text { State }\end{array}$ & Will \\
\hline \multirow{4}{*}{ Motivation } & Play $\left(w_{1}\right)$ \\
\cline { 2 - 2 } & Comradeship $\left(w_{2}\right)$ \\
\cline { 2 - 2 } & Contact Comfort $\left(w_{3}\right)$ \\
\cline { 2 - 2 } & Pain $\left(w_{4}\right)$ \\
\hline \multirow{3}{*}{ Homeostasis } & Energy $\left(w_{5}\right)$ \\
\cline { 2 - 2 } & Fatigue $\left(w_{6}\right)$ \\
\cline { 2 - 2 } & Excretion $\left(w_{7}\right)$ \\
\hline Emotion & Emotion $\left(w_{8}\right)$ \\
\hline
\end{tabular}

Each element of motivation and homeostasis has three states of high, mid and low. Emotion has five states of happy, sad, anger, fear, and neutral. Similarly, the assumed facts of context-based confabulation are considered as in Table 2. In the experiments, when, where and what were considered as contexts.

TABLE. 2 ASSUMED FACTS OF CONTEXT-BASED CONFABULATION
\begin{tabular}{|c|c|}
\hline Context & Phase \\
\hline \multirow{3}{*}{ Time $\left(c_{1}\right)$} & Morning \\
\cline { 2 - 2 } & Afternoon \\
\cline { 2 - 2 } & Evening \\
\cline { 2 - 2 } & Night \\
\hline \multirow{2}{*}{ Place $\left(c_{2}\right)$} & Bed Room \\
\cline { 2 - 2 } Object $\left(c_{3}\right)$ & Toilet \\
\cline { 2 - 2 } & Food \\
\cline { 2 - 2 } & Comrade \\
\hline
\end{tabular}

\section{Experimental Result of Behavior Selection}

Each experiment describes the result of two-step learning. At the first step, all probabilities are initialized by an expert such as developer or user. Then, reinforcement learning is conducted by interaction between user and artificial creature. As user hits or pats the artificial creature, it can give a reward or 
penalty for reinforcement learning. In other words, the behavior probability is increased or decreased based on the user's stimulus. The artificial creature is rewarded (punished) if the selected behavior is appropriate (inappropriate) to the given context. In order to accomplish the objective of context-aware artificial creature, the following four experiments were carried out.

i) If the artificial creature ate, at a location near the toilet, it was punished. In Fig. 7, probability value of $p$ (toilet|eat) is plotted as the penalty is applied. The learning process on this behavior was carried out 12 times for 52 minutes. The graph shows that it has a decreasing tendency according to user's penalty. Since the user forbade eating behavior around the toilet, the probability of the artificial creature eating there became lower.

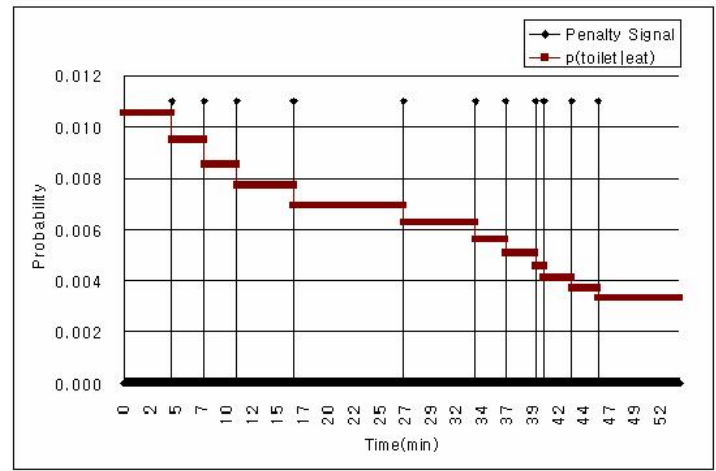

Fig. 7 Probability change when reinforcement learning is applied

Fig. 8 shows behavior frequency before and after reinforcement learning in the given context. Every result of behavior frequency was observed for 15 minutes. Only some behaviors of total 40 behaviors are plotted in the graph. The $21^{\text {st }}$ to $25^{\text {th }}$ behaviors are 'STARING', 'EATING QUICKLY', 'EATING SLOWLY', 'EXCRETING', and 'URINATING'. Before learning, the artificial creature often ate with the toilet in its vicinity. However, the frequency of eating behavior at the toilet ( $22^{\text {nd }}$ and $23^{\text {rd }}$ behavior) was reduced after reinforcement learning. Thus, the user can teach Rity proper behaviors at a certain situation by reinforcement learning.

From the next experiment onwards, behavior frequency after learning will be described in the subsequent graphs, according to the proposed two-layered confabulation.

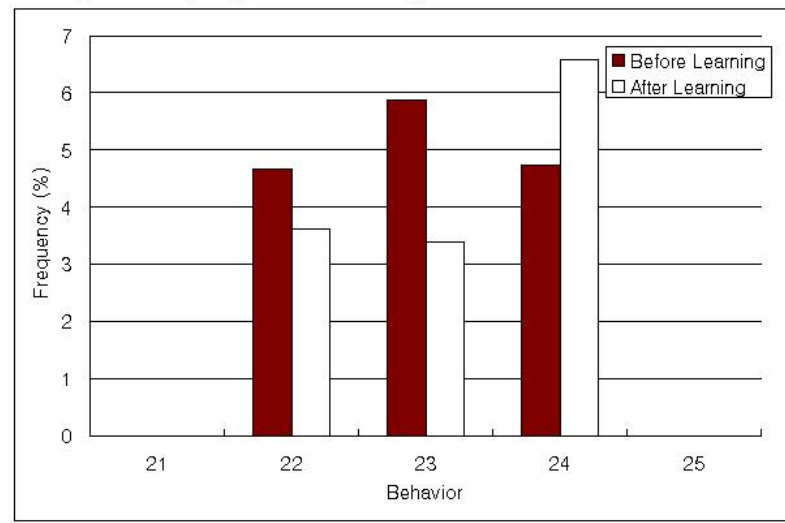

Fig.8 Behavior frequency before and after reinforcement learning for scenario (i) ii) The dominant relationship between will and context is illustrated in Fig. 9. In the experimental environment, the situation was that the creature was not hungry and context indicated that the food was in the vicinity. In this case, the creature made a decision according to the dominance of will and context. The graph shows that the eating behavior $\left(22^{\text {nd }}\right.$ and $23^{\text {rd }}$ behavior) was expressed by the effect of food in the vicinity, even though the creature was not hungry. In other words, the frequency of the eating behavior was increased only if the context was the food in the graph. This result came from both the reinforcement learning of user and the proposed two-layered confabulation architecture.

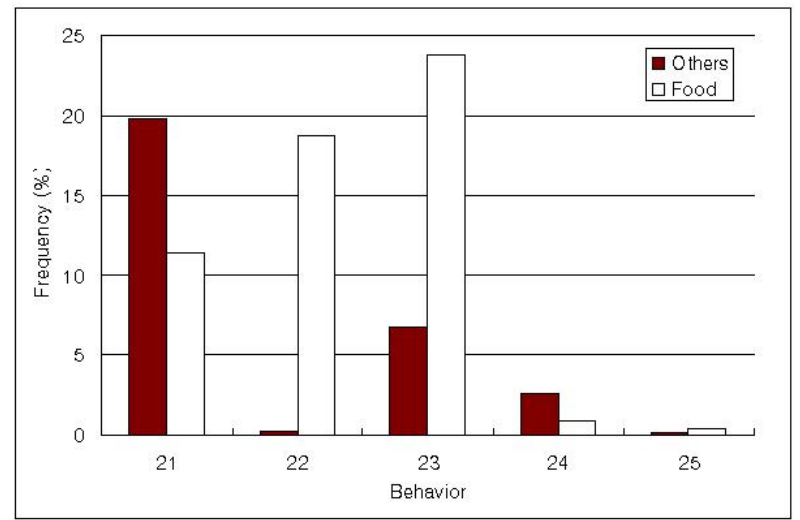

Fig.9 Behavior frequency after reinforcement learning for scenario (ii)

iii) Fig. 10 shows that the behavior related to a specific context was not selected, even though the creature has a low value of will. The $26^{\text {th }}$ to $29^{\text {th }}$ behaviors are 'SLEEPING', 'SNOOZING', 'DIGGING QUICKLY', and 'DIGGING SLOWLY'. The graph describes that under low states of sleepiness, the creature did not sleep, since the bed was not in the surroundings. However, the creature slept frequently when the bed was near.

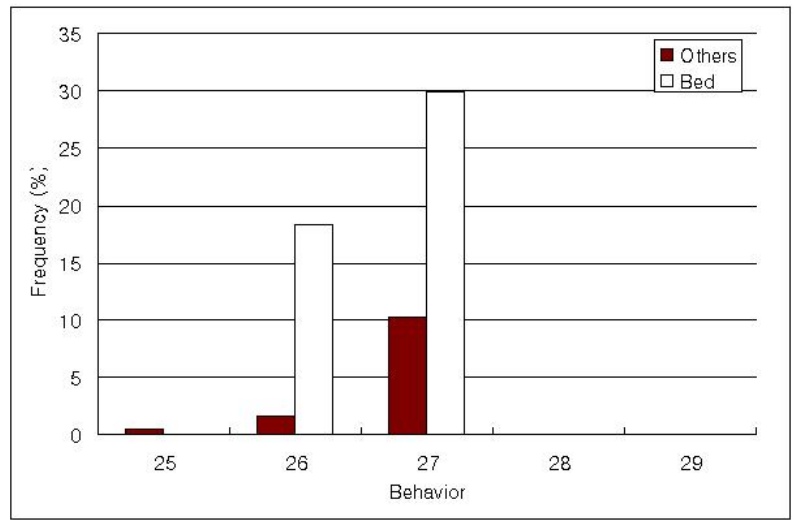

Fig. 10 Behavior frequency after reinforcement learning for scenario (iii)

iv) The behavior, which is not related to the context, would be selected if creature has a strongest will. Fig. 11 shows that the sleeping behavior was selected at all locations because the creature was absolutely sleepy. When the creature was only a little sleepy, sleeping behavior was revealed mostly on the bed. 


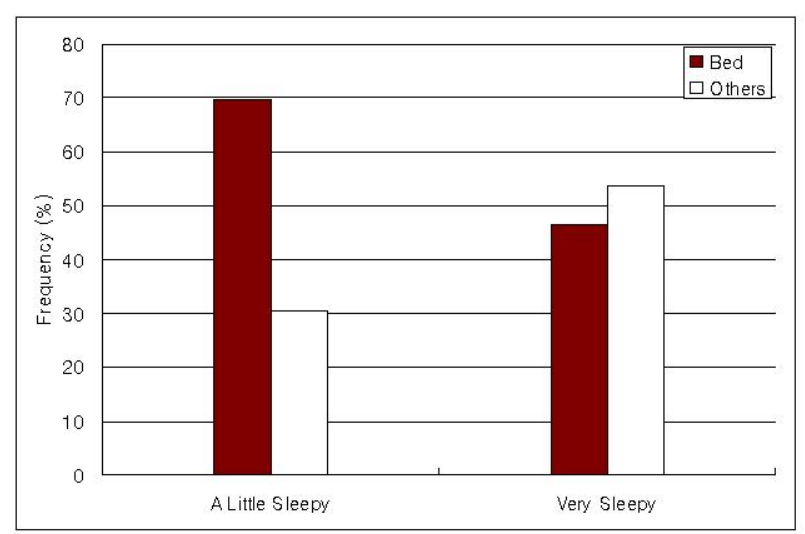

Fig.11 Behavior frequency after reinforcement learning for scenario (iv)

\section{CONCLUSIONS}

This paper proposed a novel two-layered confabulation architecture, which considers both internal state and context for the artificial creature's behavior selection. In will-based confabulation, behaviors are selected considering the internal states such as motivation, homeostasis and emotions. The selected behaviors are forwarded to the context-based confabulation to consider when, where and what. The arbiter finally decides the most fitting behavior among the suggested behaviors from the two confabulation layers.

A learning mechanism, which modifies the probabilities in memory module using user's reward and penalty inputs, was proposed in order to train the artificial creature's behaviors. The artificial creature was embodied by having real creature's characteristics using behavior sets and internal states. It could recognize the context of external environment using exteroception and its will was produced by mimicking a real creature's interoception mechanism. The experimental results showed that the artificial creature could select the most adequate behavior to meet its own desires and the context.

\section{REFERENCES}

[1] Robert Hecht-Nielsen, Cogent Confabulation, Neural Networks 18 pp. $111-115,2005$

[2] Robert Hecht-Nielsen, The Mechanism of Thought, 2006 Int. Joint Conference on Neural Networks, July 16-21, 2006

[3] Robert Hecht-Nielsen, A Theory of Cerebral Cortex, Computational Neurobiology Institute for Neural Computation, December 22, 2004

[4] N.-S. Kuppuswamy, S.-H. Cho and J.-H. Kim, "A Cognitive Control Architecture for an Artificial Creature using Episodic Memory," SICE-ICASE International Joint Conference 2006, Busan, Korea, pp. 3104-3110, Oct. 2006

[5] K. Kawamura, D.C. Noelle, K.A. Hambuchen, and T.E. Rogers, "A multi-agent approach to self-reflection for cognitive robots", in Proc. of 11th Int'l Conf. on Advanced Robotics, Coimbra, Portugal, June 30 - July 3, 2003, pp. 568-575, 2003.

[6] K. Kawamura, R.A. Peters II, D.M. Wilkes, W.A. Alford, and T.E. Rogers, "ISAC: foundations in human-humanoid interaction," IEEE Intelligent Systems, July/August 2000, pp. 38-45, 2000.

[7] K. Kawamura, W. Dodd, P. Ratanaswasd, and R.A. Gutierrez, "Development of a Robot with a Sense of Self", Proc. of the 6th IEEE Intl. Symp. on Computational Intelligence in Robotics and Automation (CIRA), Espoo, Finland, June, 2005

[8] M. Skubic, D. Noelle, M. Wilkes, K. Kawamura, and J.M. Keller, "A biologically inspired adaptive working memory for robots," AAAI Fall Symp., Workshop on the Intersection of Cognitive Science and Robotics: From Interfaces to Intelligence, Washington DC, October 2004.
[9] P. Ratanaswasd, W. Dodd, K. Kawamura, and D. Noelle, "Modular behavior control for a cognitive robot," 12 th Int'l Conf. on Advanced Robotics (ICAR), Seattle WA, July 18-20, 2005, in review.

[10] R. O'Reilly, T. S. Braver, and J. D. Cohen, "A biologically based computational model of working memory", Models of Working Memory: Mechanisms of Active Maintenance and Executive Control, (A. Miyake and P. Shah, Eds.) Cambridge; Cambridge University Press, 1999.

[11] S.-Y. Yoon, B. M. Blumberg, and G. E. Schneider, "Motivation driven learning for interactive synthetic characters," in Proceedings of Autonomous Agents, pp. 365-372, 2000.

[12] Y.-D. Kim, J.-H. Kim and Y.-J. Kim, "Behavior Selection and Learning for Synthetic Character," in Proc. of the Congress on Evolutionary Computation, Portland, USA, pp. 898-903, Jun. 2004

[13] J.-H. Kim, K.-H. Lee, and Y.9. Kim, "The Origin of Artificial Species: Genetic Robot," International Journal of Control, Automation, and Systems, vol. 3, no. 4, pp. 564-570, Dec. 2005

[14] J.-H. Kim, K.-H. Lee, Y.-D. Kim and I.-W. Park, "Genetic Representation for Evolvable Artificial Creature," in Proc. of IEEE Congress on Evolutionary Computation, Vancouver, Canada, pp. 6838-6843, Jul. 2006

[15] K.-H. Lee, C.-H.Lee, H.-L. Choi, M.-J. Tahk and J.-H. Kim, "Characterization of Artificial Creature using Multi-objective Optimization," in Proc. of Congress on Evolutionary Computation, May. 2002

[16] C.-H. Lee, Y.-J. Kim and J.-H. Kim,, "Characterization of Artificial Creature using Multi-objective Optimization," in Proc. of the 2002 FIRA Robot World Congress, May. 2002

[17] Y.-D. Kim, Y.-J. Kim, J.-H. Kim and Jong-Rak Lim, "Implementation of Artifcial Creature based on Interactive Learning," in Proc. of the 2002 FIRA Robot World Congress, May. 2002

[18] D. A.Norman and T. Shllice, "Attention to action: willed and automatic control of action," Plenum Press, New York, 1980

[19] Yi Zhang, Attention Switching During Interruptions, Proc. of the Third International Conference on Machine Learning and Cybernetics, Shanghai, 26-29 August 2004

[20] C. Breazeal, A motivational system for regulating human robot interaction, in Proceedings of the National Conference on Artificial Intelligence, Madison, WI, 1998, pp. 54-61.

[21] C. Breazeal, A. Edsinger, P. Fitzpatrick, B. Scassellati, Active vision systems for sociable robots, IEEE Transactions on Systems, Man and Cybernetics 31 (5) (2001).

[22] C. Breazeal, Designing Sociable Robots, MIT Press, Cambridge, MA, 2002.

[23] C. Breazeal, Toward sociable robots, Robotics and Autonomous Systems 42 (2003) 167-175.

[24] Cynthia Breazel, "Robot in Society: Friend or Appliance?", workshop on emotion-based agent architectures, pp 18-26, 1999

[25] N. Cowan, "The magical number 4 in short-term memory: A reconsideration of mental storage capacity" Behavioral and Brain Sciences, 24(1), 2001, pp. 87-185. 